\title{
El sistema de formación de maestros en México: Continuidad, reforma, cambio y desafíos de la política educativa ${ }^{1}$
}

\section{Teachers' education system in México: Continuity, reform, change and challenges for educational policies}

\author{
José Eriberto Cifuentes Medina \\ Magíster en Educación \\ Especialista en Educación con énfasis en Evaluación Educativa \\ Correo electrónica: joseeriberto.cifuentes@uptc.edu.co \\ Docente de la Universidad Pedagógica y Tecnológica de Colombia \\ Aura Lucía Camargo Silva \\ Magíster en Educación \\ Licenciada en Matemáticas y Física \\ Universidad Pedagógica y Tecnológica de Colombia. \\ Docente de la Universidad Pedagógica y Tecnológica de Colombia. \\ Correo electrónico: aura.camargo@uptc.edu.co \\ Universidad Pedagógica y Tecnológica de Colombia, Tunja, Colombia
}

Recibido: 20 de febrero de 2017. Aprobado: 25 de abril de 2017

Cómo citar este artículo:

Cifuentes, J.E., y Camargo, I. (2017). El sistema de formación de maestros en México: Continuidad, reforma, cambio y desafíos de la política educativa. Espiral, Revista de Docencia e Investigación, 7(2), 107 -119.

\section{Resumen}

Tipología y objetivo. El presente artículo de reflexión comparte los resultados alcanzados hasta el momento acerca del sistema de formación de maestros en México. Continuidad, reforma y cambio.

Metodología. El primero y quizás el elemento primordial de investigación corresponde a la heterogeneidad en todos los aspectos de la formación docente: administrativos, organizacionales, curriculares, profesionales y laborales; al igual que por las mismas particularidades de cada persona que elige por vocación u ocasión el ejercicio de ser docente. Como ejes de análisis en la formación docente y también en caso comparativo se pueden evidenciar de cierta manera en los demás países de América Latina.

Resultados. Dan cuenta que a través de la ilación de la disertación, se evidencian los ejes centrales de las políticas de formación docente en México que señala Aguerrondo (2003) de manera sucinta se enuncian y desarroIlan: el impacto en el presupuesto, cambios normativos para mejorar la calidad de los docentes, lograr estrategias integrales y coherentes en su proceso de enseñanza y estas decisiones pueden percibirse como amenazantes por las características mismas del sistema educativo.
Palabras clave: Formación de maestros, continuidad, reforma, cambio, desafíos, política educativa.

\section{Abstract}

Text typology. In the following reflection paper, we share partial research results about the education of Mexican teachers. Continuity, reform and change based on Arnaut (2004).

Research methodology. The first and probably the most essential element in this research is the heterogenic analysis in regards of all aspects of teachers' education: administrative, organizational, curricular, professional. We also include the reasons why each person chooses to be a teacher- be that vocational or contextual. This constitutes an analysis about the education of teachers. We also draw some comparisons with other countries in Latin-America.

Results. The results show that the main components in teachers' education policies as pointed by Aguerrondo (2003) create, through an impact in budget, some normative changes for improving the quality of teachers. This ends up in integral and coherent strategies in the teaching process; however, these decisions can be perceived as threatening because of the very characteristic of the educational system.

1 Artículo de reflexión. Se deriva del proyecto titulado: “Tendencias pedagógicas en el proceso de enseñanza y formación socio humanística y ética en estudiantes de una licenciatura en educación Básica". Código SGI 2258 otorgado por la Dirección de Investigaciones de la Universidad Pedagógica y Tecnológica de Colombia del grupo de investigación SIEK en categoría B, según Colciencias. 
Keywords: Teachers' education, continuity, reform, change, challenges, educational policies.

\section{Introducción}

Se aborda en tres partes el proceso de transformación que el sistema de formación docente ha tenido en la última década, así como las reformas y el sistema de selección e ingreso a las instituciones formadoras de docentes: a. Los distintos factores que han transformado el sistema de formación de maestros; b. Continuidad y cambio en la última década y c. Transformación de la profesión docente. En México, la Secretaría de Educación Pública es quien está a cargo de la dinamización de la educación en los diferentes estados que conforman el país y es desde esta entidad que se coordina las políticas estatales de educación con sus respectivas repercusiones políticas de los gobiernos de turno. La política integral de formación y desarrollo profesional de los docentes que se coordina desde la Secretaría de Educación Pública pretende, a través de las diversas reformas, impulsar el cambio de la mirada de los procesos de enseñanza por las razones de transformación de la sociedad actual, sin embargo, la repuesta de los docentes no es total y en algunos casos se justifica, pues se da lejos de la realidad de cada contexto.

La disertación en el marco de la diversidad de reformas educativas y de los sistemas educativos no se excluye para bien o mal a los profesores como ejes articuladores de la dinamización o rechazo de las políticas educativas establecidas.

En el estudio de Aguerrondo (2003) y que coincide con Avalos (1999) y quien asegura que, en el marco del cambio de los sistemas educativos, una condición fundamental es que los profesores puedan cambiar, por dos razones principales: por un lado, deben hacerlo si queremos que los estudiantes desarrollen nuevas formas de aprendizaje; y por el otro, porque si los profesores no acompañan y apoyan las reformas, los más hermosos diseños fracasarán (Avalos, 1999).

Los países de América Latina, desde la Independencia y en la conformación de República, han contado con diversos sistemas educativos; como consecuencia de los intereses de los gobiernos de turno y los principios políticos pocos de ellos han acertado y concertado las reformas de acuerdo con las necesidades de los educandos, educadores y de la sociedad. La formación docente genera desafíos en medio de la política educativa reformista, como corresponde ahora el análisis a México y que coincide de cierta manera con los países latinoamericanos.

Al igual se hace un análisis hermenéutico de: los condicionantes de las decisiones sobre formación docente en el marco de las reformas educativas, desafíos relativos a cómo mejorar la formación docente inicial, mejorar la calidad de los docentes en servicio y las modificaciones en la carrera docente. En el arte de disertar y destacar los ejes centrales de las políticas de formación docente en México que señala Aguerrondo (2003) en el impacto en el presupuesto, cambios normativos para mejorar la calidad de los docentes, lograr estrategias integrales y coherentes en su proceso de enseñanza y estas decisiones pueden percibirse como amenazantes por las características mismas del sistema educativo.

El análisis del sistema de formación de maestros en México, como continuidad, reforma, cambio y desafíos de la política educativa es una investigación que varios autores han realizado al respecto, pero que siguen siendo objeto de estudio y disertación, en la medida en que el tema recobra vigencia real y hace parte del plan de gobierno actual y de seguro de posteriores. La relación de la educación con los maestros es directa y de consecuencia directa, pues se trata de la relación de maestros y educandos en el proceso de enseñanza y aprendizaje. 


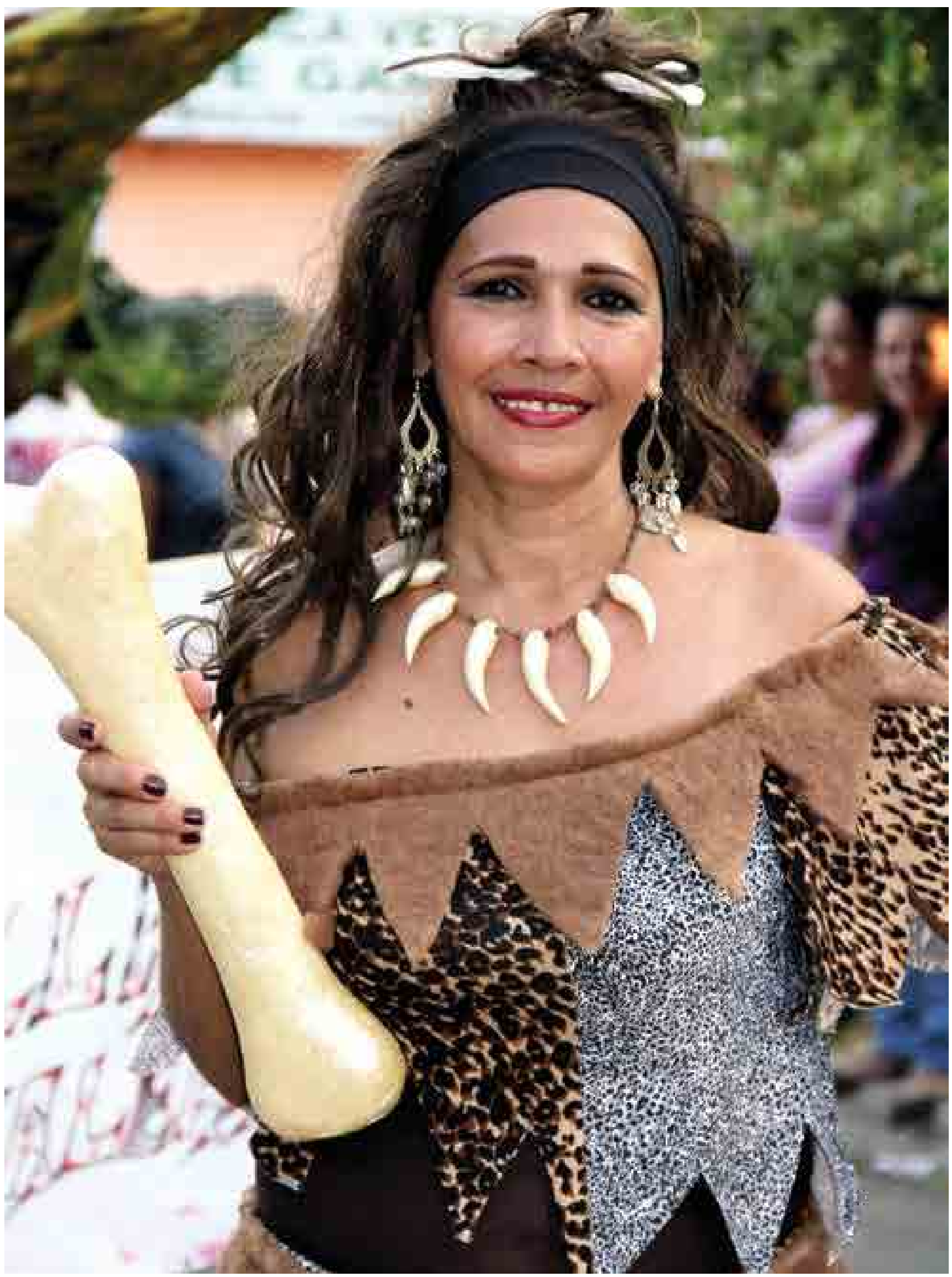




\section{Los distintos factores que han transformado el sistema de formación de maestros}

La educación en América Latina ha sido influenciada por la globalización de sobremanera, teniendo en cuenta que los países latinoamericanos han copiado, acomodado e importado modelos educativos de países anglosajones $y$, especialmente europeos, incluso a las reformas en la formación docente que allí surgen, también se hacen en nuestros países, por ello

La transformación de la educación en una de las más importantes inversiones requirió, a su vez, cambiar la definición tradicional que se tenía de ellas: en adelante se concibió la educación como una empresa que debía cumplir con ciertos niveles de rendimiento financiero y que debía verificar sus procesos, con el objeto de optimizar la aplicación de recursos económicos" (Ordoñez, 1995).

Los nuevos énfasis y exigencias del mundo moderno impusieron a la formación docente una reorientación de sus objetivos y procedimientos, a través de diversas reformas, al igual que para asignarle una nueva identidad social, vinculándola al desarrollo en todas las actividades atinentes al orden de la producción y calidad de vida de la comunidad educativa. Refiriendo a la escuela como un sistema de producción en relación con el crecimiento cuantitativo y de medición; como consecuencia de los efectos de la globalización y de los cambios globales que se aplica a los docentes en ejercicio y a quienes ingresan al servicio docente, y la forma como a educación se ha venido convirtiendo en proceso de mercantilización, educación para la renta como bien lo señala Nussbaum (2010).

En la perspectiva de Ordoñez (1995) señala que, "durante las tres décadas, a pesar de que frecuentemente se hicieron reformas y se diseñaron nuevos programas, el sistema educativo creció en rigidez e inmovilismo y aumentó entre las comunidades el escepticismo respecto de sus posibilidades de transformación" (p. 30). Interesa a los gobiernos proponer en las reformas educativas: la cobertura y la calidad, pero la segunda dependiente y abrumada por la primera, sobrecargando a los docentes. La cuestión conlleva indicar que se ha de prestar atención al problema de la transformación cualitativa, mediante la adecuación de los tópicos de aprendizaje a las características culturales de las comunidades a las que se presta el servicio educativo por parte de los maestros.

En la perspectiva de Arnaut (2004), en relación con la calidad del servicio docente, están a la cabeza las instituciones formadoras de maestros, iniciando por las normales, que no han permanecido inmunes a los cambios, sino también han sido objeto de reformas por las políticas internas y externas, para influenciar los diferentes escenarios: social, cultural, político, entre otros, entre tanto se hace necesario referir la obra coordinada por Hernández (2015) que se denomina Educación y narrativa, Voces y vivencias de los profesores de Chiapas y Oaxaca. De la formación a la acción es todo un reto para el maestro, y su experiencia la construye y la edifica con los contenidos y las dinámicas del contexto de la comunidad educativa.

Las transformaciones en la formación de maestros en México en las últimas décadas, ha optado por la transversalidad en las transformaciones de acuerdo a la política educativa, y derivado del contexto social, cultural y político. Lo más probable es que la segunda y la tercera están determinadas por las políticas de estandarización de la educación que más cosas negativas lleva consigo.

\section{Continuidad y cambio en la última década}

En esta perspectiva Arnaut (2004) argumenta que "muchas tensiones y tendencias al cambio vividas por el sistema de formación de maestros desde la década de los 70 se fueron acentuando al iniciar la década de los 90, debido a una serie 
de reformas político-administrativas y curriculares"; a nivel de la educación básica y del propio sistema de formación de maestros en México.

Tabla 1. Continuidad y cambio en la última década

ASPECTO

Reforma políticoadministrativa

Reforma curricular de la enseñanza normal

Desarrollo de la formación permanente del magisterio

\section{BREVE DESCRIPCIÓN}

En 1992 el gobierno federal transfirió a los gobiernos de los estados todas las escuelas normales que hasta entonces estaban bajo su dependencia. La Ley General de Educación de 1993 reservó como atribución exclusiva del gobierno federal la facultad de determinar para toda la República los planes y programas de estudio para normal y formación de maestros de educación básica.

En 1996 la SEP, en coordinación con las autoridades educativas estatales, puso en marcha el PTFAEN, que comprendía la reforma curricular de las licenciaturas que ofrecen las escuelas normales para todos los niveles y modalidades de la educación básica. En 1997 entró en vigor un nuevo plan de estudios para la Licenciatura en Educación Primaria; en 1999 los planes de las licenciaturas en Educación Preescolar y Secundaria; en 2002 el de la Licenciatura en Educación Física, encontrándose en proceso la renovación de los planes de las licenciaturas en Educación Especial, Artística y para el Medio Indígena.

La conducción y la gestión de los programas de formación permanente de maestros de educación básica presentan las mismas dificultades que ya se señalaron para el subsistema de formación inicial y otras más. Incluso se puede afirmar que la empresa de transformar y consolidar los programas de formación permanente se antoja más compleja, ardua y plagada de dificultades, que la referida a la formación inicial del magisterio.

Fuente: Información tomada de Arnaut, 2004.
La historia es necesaria, y para el caso objeto de estudio, valida la confrontación en línea de tiempo que acompañada de la normatividad, se pueden visualizar las reformas desde el contexto nacional en el orden de la formación de maestros; acompañadas por la reforma curricular de enseñanza y el desarrollo necesario de la formación de los maestros antes del ingreso y luego en el servicio docente. La historia permite presentar las reformas de las últimas décadas $y$, desde luego, a las demás ciencias les corresponde el análisis de impacto para determinar el cambio y las transformaciones.

\section{Transformación de la profesión docente}

En México en la última década se ha evidenciado la diversidad de la profesión docente, como resultado de los cambios que iniciaron en la década de los 70, fortalecidos con la federalización en 1992 y la Ley General en 1993. Las modificaciones son significativas en el salario, las condiciones de trabajo, las reformas curriculares, la implementación de programas de formación y desde luego la revolución tecnológica, entre otros cambios de orden sociodemográfico que impactan la realidad de la formación de maestros entre finales del siglo $X X$ y los inicios del siglo XXI. 


\section{Tabla 2. Transformación de la profesión docente}

ASPECTO

Salario profesional y Carrera Magisterial

Reforma curricular y otros programas educativos

Hacia una mayor diversidad y mixtura del magisterio

\section{BREVE DESCRIPCIÓN}

La federalización educativa de 1992 y la Ley General de Educación de 1993 reservaron y en cierto sentido reforzaron las atribuciones del Gobierno federal en la determinación de las normas, la organización y los contenidos de la educación básica y normal y del resto de las instituciones formadoras de maestros de educación básica.

La dramática caída de los salarios reales del magisterio en servicio y la esclerosis de la pirámide profesional dentro del antiguo sistema escalafonario recibieron como respuesta, en 1989, la promesa de establecer un salario profesional para este sector, y el establecimiento de la Carrera Magisterial como un escalafón paralelo que estimulara los esfuerzos de los docentes en su formación y en el desempeño de sus funciones.

La reforma curricular de la educación básica, la progresiva diferenciación curricular y la puesta en marcha de diversos programas encaminados a mejorar la calidad y la equidad educativas plantearon la necesidad de crear, expandir y diferenciar los cuadros de carácter técnico pedagógico, tanto en la administración educativa federal como en las nuevas administraciones educativas de los estados, así como en las oficinas centrales y en las instancias más cercanas a las escuelas, como la supervisión escolar y dentro de las escuelas mismas.

Las sucesivas reformas a los planes y programas de enseñanza normal, y la progresiva diversificación de la oferta curricular del sistema de formación inicial y permanente del magisterio ha traído consigo una acentuada tendencia hacia la diferenciación del perfil profesional del magisterio. El magisterio en servicio se ha diversificado como resultado de la progresiva diferenciación curricular de los tres niveles de la educación básica. En fin, el magisterio ya no es lo que fue o, mejor dicho, ya no solo es lo que había sido.

Fuente: Información tomada de Arnaut, 2004.

En este sentido, Aguerrondo (2002) señala que, "el ingreso, permanencia y ascenso en la carrera laboral de los profesores tienen características claras que condicionan la organización del trabajo en educación". En la medida que las reformas contribuyan con la preparación de los docentes que están en carrera y propugnen por la formación continua en el ahora y para el futuro, se garantizará la calidad educativa.

\section{Los condicionantes de las decisiones sobre formación docente en el marco de las reformas educativas}

El sistema de reformas educativas que se han desarrollado durante décadas y que ha dedicado espacio a los diferentes actores de la comunidad educativa, centra ahora su atención en los profesores. No se constituye entonces esen- cia ontológica de si son culpables o inocentes de los problemas de la educación actual, pero sí son los actores que tienen que jugar un papel protagónico en medio de la dinamización o rechazo de las reformas de los sistemas educativos si entre ellos no se ha dado la apuesta por la concertación para la elaboración y puesta en marcha de las transformaciones educativas, y si por el contrario se han impuesto a la fuerza que no conduce a la verdad de una reforma valida y acertada, que en tiempo se podrá ver los resultados del impacto causado.

En este sentido, Aguerrondo (2003) sostiene que:

los modelos de formación de profesores, las condiciones de empleo y de trabajo de la gran mayoría, su inserción subordinada en la organización educativa, la calidad y orientación de su 
formación, la rigidez y uniformidad del curriculum, son algunos de los elementos que resisten los posibles cambios (p. 10).

Por lo tanto, emergen cuatro ejes centrales acerca de los docentes en el marco de las reformas educativas de México y de los países latinoamericanos. El impacto en el presupuesto, cambios normativos para mejorar la calidad de los docentes, lograr estrategias integrales y coherentes en su proceso de enseñanza y estas decisiones pueden percibirse como amenazantes por las características mismas del sistema educativo.

El reto del Estado y de las reformas educativas se centra en cómo mejorar el desempeño docente y por ello plantea diversidad de iniciativas que se traslapan en los desafíos que entrañan los ejes de las políticas de formación docente. Así, los retos se dan en relación con la calidad con la que enseñan y en las condiciones de trabajo en que enseñan en aras de un avance por una educación de calidad.

La puesta en marcha de las reformas a los sistemas educativos en la medida que afecte a los docentes será un factor de tensión su implementación, por falta de concertación u otras razones que, al considerar la actualidad de las reformas en América Latina, se hace compleja la disertación, en la medida que está afirmando en plural: las reformas y es de igual manera plural los países de Latinoamérica. En los cambios planteados para consolidar los sistemas educativos, se discute la mejora en los indicadores de calidad, y afirma de forma cuantitativa la cobertura de la educación pública, casi que sometiendo a los profesores a las reformas establecidas.

En la preocupación de los gobiernos reformistas en los diferentes campos de la sociedad, no ha sido una excepción la educación en el interés se mostrar la superación de índices de alfabetización y el ingreso a la educación pública han descuidado en ciertas ocasiones la calidad de esta y el trabajo de los profesores. En
América Latina se han organizado equipos de planificación educativos, cuyas obras señalan la conveniencia de los proyectos educativos para mejorar la gestión de los planteles, con el fin de mejorar los resultados educativos de los países, por tanto, Ordoñez (1995) afirma que:

tal es el caso de Venezuela y Ecuador donde, con el apoyo del Centro Interamericano de Estudios e Investigaciones para el Planteamiento de la Educación, se organizaron equipos para participar en el Programa de Formación de Recursos Humanos en Gerencia Educativa (p. 32).

Las reformas a la educación se han realizado en todos y cada uno de los países de América desde México hasta Chile; cada uno con sus dinámicas propias de desarrollo histórico y con la diversidad de influencias de modelos educativos de países vecinos y lejanos. En este sentido, Paladines (2004) sostiene que, Ecuador cuenta con su propia historia de la educación y el pensamiento pedagógico en el siglo XIX, desde los mismos procesos de independencia como punto coyuntural de cambios en todos los campos de los países latinoamericanos, elaborando en la línea del tiempo una periodización: de la Colonia a la República y la conformación del Estado Nacional en lo referente al siglo XIX.

En el siglo XX las reformas en Ecuador han sido significativas, al respecto Paladines (2004) así lo considera en la investigación titulada: Historia de la educación y el pensamiento pedagógico ecuatorianos: Siglo XX. Se ocupa de los antecedentes y la constitución del Estado moderno, de la polémica entre la educación católica y la educación laica, el paso y las crisis de la educación laica. Son ejemplos de algunos de los países de América Latina en el Estado de las reformas de Estado implementadas en cada país con resultados diferentes por la posición de contextos e intereses generales y particulares de gobernantes, profesores, educandos y de la sociedad en general.

México es uno de los referentes de mayor importancia en la educación y desde luego en 
las reformas al sistema educativo, se resalta la investigación de Galván (2004) La educación mexicana en el siglo XIX: 1821 - 1921, él divide su estudio en tres periodos: 1. Los primeros pasos 1821-1867; 2. Hacia la formación del sistema educativo mexicano 1867-1910 y 3. La educación y el movimiento revolucionario 1910-1921. Comienza en los procesos de independencia con un estado liberal y su desarrollo sistemático con sus diversas reformas al sistema educativo y termina en 1921 con la creación de la Secretaría de Educación Pública en medio de un proceso de reestructuración al sistema educativo mexicano.

Los proyectos educativos en México 19211982 es una investigación de Lazarin (2004), la cual está dividida en periodos: 1. La educación pos-revolucionaria 1921-1940; 2. El milagro económico y la educación para la unidad nacional 1940-1968 y, 3. La crisis económica, la globalización mundial y los nuevos modelos educativos 1968-1988. Denotan la importancia de las reformas educativas en México a lo largo de dos siglos, y consolidan la dinámica activa y el progreso de a la educación en este país.

\section{Desafíos relativos a cómo mejorar la formación docente inicial y docentes en servicio}

Considerar en la formación de profesores un aspecto de especial relevancia para mejorar la calidad de la profesión, las estrategias en la enseñanza y el interés por la cualificación permanente serán de especial impulso por la motivación en la formación inicial, es decir, en el proceso académico de estudio en las escuelas normales y en el nivel de pregrado. La formación docente inicial asegura Aguerrondo (2003) y (2002) es fundamental para redefinir la tarea de enseñar, promover cambios profundos y transformaciones en la educación y en el proceso didáctico de la enseñanza. Por lo tanto, es necesario un sucinto análisis de los retos y desafíos en este escenario.

\section{Tabla 3. Desafíos en la formación docente inicial}

\section{DESAFIO}

Los cambios en la estructura y contenidos de la formación docente inicial

El control de la calidad de la formación que se imparte

El reordenamiento de la oferta de instituciones formadoras de profesores

\section{BREVE DESCRIPCIÓN}

En América Latina la respuesta ha sido diversa, aunque la tendencia predominante es trasladar la formación inicial al nivel universitario o al nivel superior no universitario. En la mayoría de los países las viejas escuelas normales han sido transformadas en institutos superiores con carreras que van de dos a cinco años, y cuyo requisito de ingreso es que se haya completado el secundario.

Las intervenciones políticas en estos temas se enfrentan con problemas tales como la autonomía de las universidades, o la tradición de las instituciones terciarias, poco habituadas a funcionar con controles de calidad externos que permitan dar cuenta de su competencia académica.

Un tema importante en relación con los desafíos a encarar es precisamente la necesidad de acuerdos de reordenamiento de la oferta de formación docente dentro de las distintas regiones del país, problemática que sobrepasa la mera mirada institucional. Es necesario tener en cuenta también la oferta universitaria, y pactar con estas instituciones una distribución más racional de los esfuerzos.

Fuente: Información tomada de Aguerrondo, 2003. 


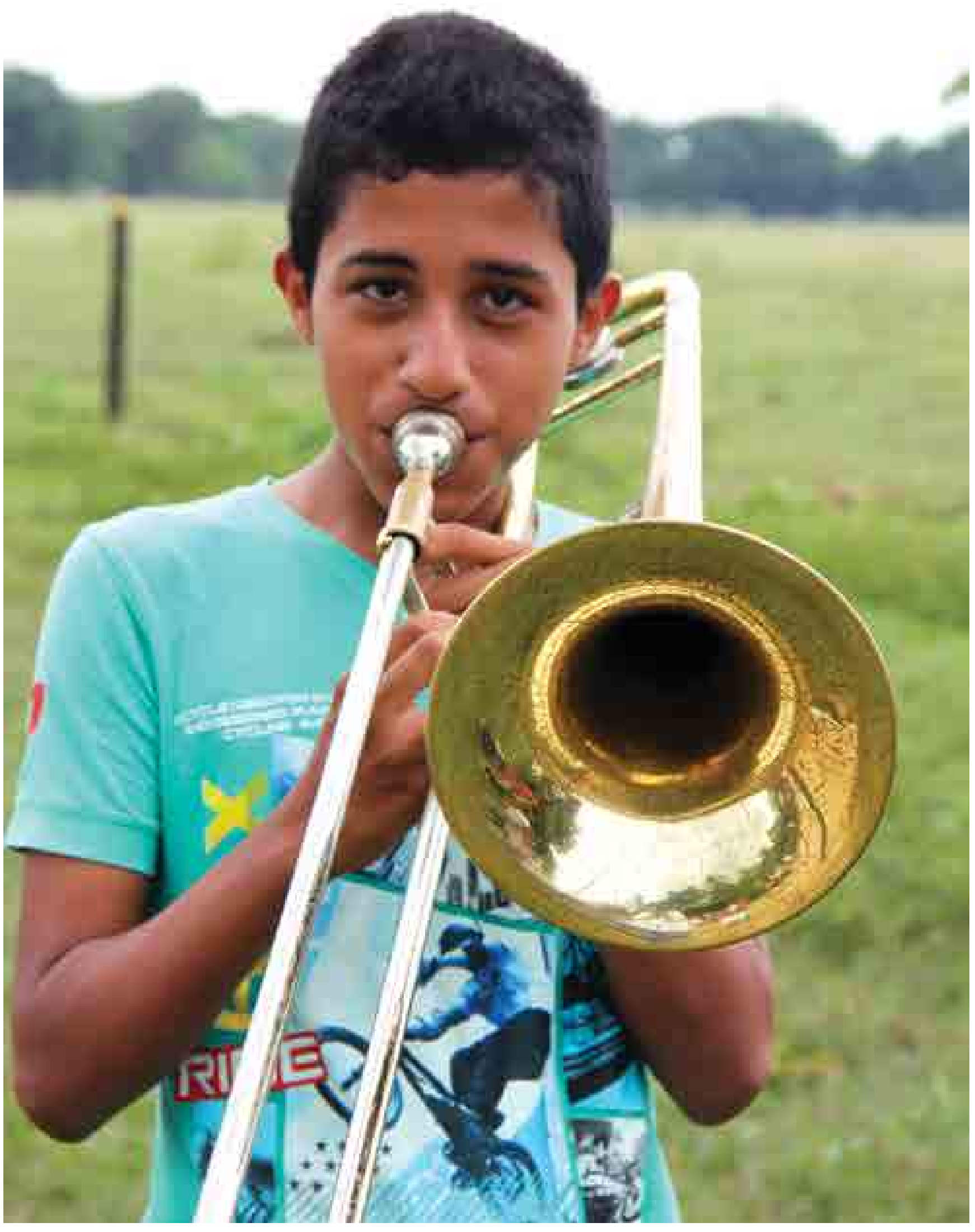


Los desafíos planteados son derivados de estudios y elevados a la práctica a través de las reformas de los sistemas educativos que para el caso objeto de la disertación, refiere a la educación inicial que reciben los profesores en y desde la universidad para su posterior ejercicio en la docencia, en los diferentes niveles educativos y que, desde luego, es necesario y pertinente fomentar las bases de una formación y consolidación de la disciplina en estudio para motivarlos en la innovación, indagación permanente, investigación y formación continua para dinamizar su quehacer docente.

Los retos que desde el Estado han de afrontar los docentes para que haya interés en su formación continua, no como imposición sino como necesidad en la transformación continua de su práctica docente en aras de oxigenar su praxis pedagógica, refrescar los contenidos disciplinares e interdisciplinares para innovar, indagar e investigar y darle sentido significativo al proceso de enseñanza para el bien de la comunidad educativa.

El profesor en ejercicio requiere partir del hecho de ser persona, si no lo es; difícilmente puede ayudar a la formación de gente comprometida con la institución y con la solución de los problemas que requiere su contexto. Surge la propuesta de Recio (2011) al respecto de las dimensiones y cualidades que ha de desarrollar un docente, una primera dimensión corresponde a la formación integral como persona y

Se podrían agregar otras como autoestima, dignidad, hacer uso correcto de la libertad, capacidad de tomar decisiones, seguir sus propios criterios, honestidad, ética, capacidad de escucha, apertura, mente abierta, respeto por las ideas ajenas, tolerante, compañero del proceso de aprendizaje del alumno y exige, pero dando mucho de sí mismo (Recio 2011).

Las dimensiones de un profesor, propuestas por Recio (2011), aplican para un docente en cualquiera de los niveles de educación en la medida que ha de ser una persona integral, ha de tener dominio por un saber específico, ser un pedagogo, ejercer a cabalidad la docencia, un buen investigador e infundir este propósito en sus estudiantes, orientar a sus alumnos por el buen camino, mantener buenas relaciones interpersonales con ellos, también con sus compañeros y los directivos, amor y vocación por su profesión, un gran visionario y excelente líder en pro de sociedad justa, educada y con excelentes profesionales.

Según Lozano (2011) el verdadero y auténtico profesor ha de ser prudente y sabio, en el ejercicio de su labor ha de estar asistido por cualidades de persona integral y le es necesario desarrollar en equilibrio sus dimensiones, para brindar un servicio de calidad a sus estudiantes.

\section{Desafíos relativos a las modificaciones en la carrera docente}

El trabajo de competencia estatal en el caso de México y de los demás países latinoamericanos en relación con las modificaciones en proyección, las reformas que puedan surgir y la preparación para los docentes que están en carrera son necesarias desde la formación continua en el ahora y futuro, al igual que en diversos aspectos de su desempeño laboral, como lo es el factor salarial, como aquel no solo se constituya como eje de enseñanza, sino que por el contrario sea un valor agregado a su compromiso, su vocación y la calidad en su enseñanza. 
Tabla 4. Desafíos en la carrera docente

DESAFIO

1. Los profesores como servidores públicos

2. Rasgos de la carrera docente

3. Los actuales desafíos

\section{BREVE DESCRIPCIÓN}

En la región latinoamericana la educación es un servicio público, lo cual no significa que en todos nuestros países no haya una red importante de educación privada. Sin embargo, su condición de servicio público garantizado por el Estado implica que esta actividad no puede realizarse privadamente si no está enmarcada en el formato concreto provisto por el Estado, y si este no la autoriza.

El ingreso, permanencia y ascenso en la carrera laboral de los profesores tienen características claras que condicionan la organización del trabajo en educación.

Frente a la caída de la calidad de la educación, desde muchos sectores se plantea la necesidad de introducir mecanismos de rendición de cuentas en los sistemas educativos. Y esto no solo en función de la mayor eficiencia en la gestión, sino también en función de la necesidad de mayor transparencia que hoy requiere el manejo de los asuntos públicos. La discusión sobre la carrera docente es difícil porque significa en realidad discutir implícitamente todas las variables centrales que organizan la educación, esto es: el modelo de institución escolar a que se apunta, las definiciones centrales del papel del docente, la formación y la capacitación para el desarrollo profesional, los modelos de gestión de la educación en general y, también en particular, lo relativo al reclutamiento, el ingreso, el ascenso y el retiro del personal.

Fuente: Información tomada de Aguerrondo, 2003.

Los desafíos en las modificaciones de la carrera docente no es solo en México sino en los países de América Latina, y por lo tanto se evidencian factores y elementos en común. Los profesores al ingresar a la carrera docente por méritos o por razones particulares se constituyen en servidores públicos $y$, por ende, asumen las implicaciones pertinentes en todos los aspectos y escenarios.

Los docentes en ejercicio cuentan con los beneficios normativos y legales vigentes, a medida que avanzan en el tiempo adquieren antigüedad para régimen fiscal en cuanto a salario y presupuesto se refiere $y$, desde luego, en las estrategias de enseñanza, también dado a que la experiencia hace al maestro y con el apoyo de la formación continua dinamiza su práctica pedagógica disciplinar y se fortalece su quehacer y vitaliza los contenidos y el ejercicio de su enseñanza.

En el estudio realizado por el Instituto de Estudios Educativos y Sindicales de América que se denomina: ¿De dónde vienen y a dónde van los maestros mexicanos? La formación docente en México, 1822-2012 refiere que:

El reto está en los alumnos que actualmente cursan la Licenciatura en Educación y los profesores que ya se encuentran frente al aula -en cualquiera de los niveles educativos- para que logren no solo mediar, sino superar las problemáticas actuales. Que ante el bajo rendimiento académico, la deserción escolar y las críticas puedan hacer una alianza con las TIC, los padres de familia y la sociedad en su conjunto, logrando diluir el dualismo al que se enfrentan los profesores de salvadores y culpables de la calidad educativa, invitando a un proceso de autocrítica y auto reflexión profesional y gremial que permita integrar acciones educativas tendientes a la superación de los maestros del país. Obligando a reconocer que la responsabilidad de la educación no sólo se encuentra depositada en la escuela y el profesor, sino en toda la sociedad en conjunto (IEESA).

Los desafíos y retos para los maestros en formación y en ejercicio son permanentes y de 
cada día, derivados de las tensiones normativas que emanan del Gobierno y también de las tensiones de cada comunidad educativa al igual que de aquellas que emergen de los cambios de la sociedad.

\section{A manera de conclusión}

El sistema de formación de maestros en México, coordinado desde la Secretaría de Educación Pública, tiene como política integral la formación y el desarrollo profesional de los maestros de los diferentes niveles de educación: primaria, básica y superior. Los procesos de reforma impulsados en las últimas décadas influenciados en parte por la globalización y los organismos multilaterales con el caso de la OCDE, de la cual hace parte México en cierta medida contribuyen al cambio en la mirada del quehacer docente, pero se incomoda o cuando se sale del estado de confort o cuando se está haciendo una propuesta lejos de las realidades: social, económica, educativa y las demás de la formación docente.

El sistema de selección e ingreso al servicio docente en las últimas décadas ha generado controversia y se ha implementado en 6 de los 32 estados de México en el $20 \%$. Sin duda son reformas argumentadas y desde luego buscan la transformación del ingreso al servicio docente en aras de la cualificación constante y la expectativa de los docentes "que busca fortalecer la gestión, organización y vida académica de las instituciones formadoras de maestros, así como la capacidad de conducción, planeación y coordinación de los sistemas estatales de formación de maestros, el inicio de la descentralización de los talleres" (Arnaut, 2004, p. 20).

En el amplio espectro de reformas recientes en los sistemas educativos de América Latina el campo docente no ha sido la excepción y en el caso de México cuatro ejes centrales se evidencian: el impacto en el presupuesto, cambios normativos para mejorar la calidad de los docentes, lograr estrategias integrales y coherentes en su proceso de enseñanza y estas decisiones pueden percibirse como amenazantes por las características mismas del sistema educativo.

La política pública en educación, deja entre el dinamismo propio de su desarrollo de quienes: la proyectan, la estudian, aprueban e implementan todo un ciclo de desconocimiento de lógicas propias del contexto nacional y en medio de absurdos puestos en marcha se adhiere la importación de modelos educativos de otros ambientes que poco o en nada se ajustan a la realidad de cada país. Casi o escaso estudio de diagnóstico de realidades se evidencia más la imposición se evidencia para la implementación de la política que se considera es publica, de todos y para todos.

De la formación de los profesores se hace evidente por parte de la Escuelas Normales de Educación Superior y que de orden Federal, Estatal y también hay privadas y también la Universidad Pedagógica Nacional (UPN), organismo descentralizado de la SEP, forma maestros de educación básica en diversas especialidades con categoría de licenciatura, y 'nivela' a maestros que no tienen ese título. Para fortalecer su desempeño de calidad en cada uno de los niveles de enseñanza.

\section{Referencias}

Aguerrondo, I. (2002). La calidad de la educación: Ejes para su definición y evaluación. Organización de Estados lberoamericanos.

Aguerrondo, I. (2003). Formación docente: desafíos de la política educativa. Cuadernos de discusión 8. México.

Arnaut, A. (2004). El sistema de formación de maestros en México. Continuidad, reforma y cambio. Cuadernos de Discusión $N^{\circ}$ 17. Secretaría de Educación Pública, México.

Galván, L. (2004). La educación mexicana en el siglo XIX: 1821-1921. En Zuluaga, O. y Ossenbach, G. et al. (Comps.) Génesis y desarrollo de los sistemas Educativos

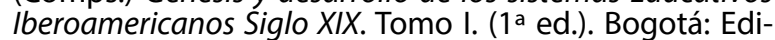
torial: Magisterio.

Hernández, A. (2015). Educación y narrativa, voces y vivencias de los profesores de Chiapas y Oaxaca. Chiapas, México.

Lozano, R. A. (Coord.). (2005). El éxito en la enseñanza: Aspectos didácticos de las facetas del profesor. ( $1^{\text {a }}$ ed.). México: Editorial Trillas. 
Nussbaum, M. (2010). Sin fines de lucro. Por qué la democracia necesita de las humanidades. Buenos Aires: Katz Editores.

Ordoñez, C. (1995). Administración y desarrollo de comunidades educativas. Hacia un nuevo paradigma de organización escolar.

Paladines, C. (2006). Historia de la educación y el pensamiento pedagógico ecuatorianos: siglo XIX. Loja, Ecuador: Universidad Técnica Particular de Loja.

Roa, K. (2015). El sistema de formación docente en México y su impacto en la Educación. Recuperado de https://prezi.
com/qgdlfdvyxg6l/el-sistema-de-formacion-Docenteen-mexico-y-su-impacto-en-la/

Secretaría de Educación Pública (2003). Hacia una política integral para la formación y el desarrollo profesional de los maestros de educación básica. Cuadernos de Discusión 1. México, D. F.

Tedesco, J. (1999). Profesionalización y capacitación docente. Unesco, Instituto Internacional de Planeamiento de la Educación. Buenos Aires, Argentina. 